\title{
Predicting hand numerical scoring of vibration exposure using an artificial neural network
}

\author{
N. A. Azmir ${ }^{1}$, M. I. Ghazali ${ }^{1}$, M. N. Yahya ${ }^{1}$, M. H. Ali ${ }^{2}$ \\ \& J. I. Song ${ }^{3}$ \\ ${ }^{1}$ Faculty of Mechanical and Manufacturing Engineering, \\ Universiti Tun Hussein Onn Malaysia (UTHM), Malaysia \\ ${ }^{2}$ Consultation, Research and Development Department, \\ National Institute of Occupational Safety and Health (NIOSH), Malaysia \\ ${ }^{3}$ Changwon National University, South Korea
}

\begin{abstract}
Ergonomic researchers are investigating the cause and effect relationship due to hand transmitted vibration exposure. These relationships make occupational safety and health practitioners determine the possible root or intermittent cause which can be harmful to workers using an artificial intelligence system. In maintenance of the agriculture sector, there is a need to study hand-arm vibration exposure due to significant vascular and sensori-neural disorder. This paper presents the development of a hand numerical scoring prediction model among grass cutter workers in Malaysia using an artificial neural network. In this study, the independent variables consist of age, working experience and estimated hand-arm vibration exposure A(8) while the dependent variables is hand numerical scoring for each hand. Artificial neural network architecture has been developed and applied to the randomized onsite data collection. The artificial neural network will be based on the feed forward back propagation network using "tan" sigmoid activation function. The results of predicting the hand-arm vibration effect were evaluated by using mean square error and regression for both hands. Both hand prediction models were produced less than 0.1 relative error of prediction as compared to actual data. Our conclusion has been that the developments of prediction model have archived the best validation and more efficient means using nonlinear techniques, hence providing health welfare among grass cutter worker against hand-arm vibration syndrome.

Keywords: hand transmitted vibration, hand numerical scoring, artificial neural network, grass cutter workers.
\end{abstract}




\section{Introduction}

Vibration is a common potential of harmful activity to worker whom using vibrating equipment in their routine job. Vibration hazard ability to cause both acute and chronic effects based on the amplitude and frequency of the vibration exposure. In occupational vibration, the intensity of the vibration consists of 3 types which are motion sickness, whole body vibration and hand-arm vibration. These types were differentiating by the frequency of exposure and where the location of vibration source transmitted to worker body. The vibration or shock transmitted energy will cause health effect due to resonance or disturbance with human vibration body. The hand-arm vibration can cause vascular and sensorineural disorder to workers hand [1]. Nerve related disease occurs when work is done repetitively, resulting in exposure of the nerve to pressure from vibrating equipment [2]. Hence, effects of numbness and pain will be felt for a period of time. Meanwhile, vascular diseases are due to the pressure of nerves and veins resulting in reduced blood flow to the muscle, tendons and ligaments [3]. So, results in slow recovery of the muscle and limited duration of muscle use.

The hazard of hand-arm vibration depends on several factors, 1) type of machine used, 2) the machine operation procedure, 3) type of personal protective equipment and, 4) type of working sector or application. Although, there are many techniques practice by safety and health practitioner on how to determine the hand-arm vibration disorder, the hand numerical scoring (HNS) is the simplest approach to identify the effect of hand-arm vibration exposure. It uses the observation and professional judgment techniques to identify any colour change of worker finger's phalanges. Each of the fingers phalange has its own numerical value and the value is accumulating for the both hand. The minimum value is " 0 " while the maximum value is " 33 " for each hand followed Stockholm Workshop Scale [4]. This technique has been proposed by department of occupational safety and health (DOSH), Malaysia under the occupational vibration exposure guideline to investigate the health effect of hand held vibrating equipment [5].

Researchers throughout all over the world have done various studies in observing the effect of hand-arm vibration hazards to the hand palm of workers. However, there are disagreement among works mainly due to bias from uncontrolled exclusion and inclusion criteria (working environment, experience involve with vibrating equipment, age, hand transmitted vibration) [6, 7]. Therefore, a health practitioner should investigate the hand-arm vibration syndrome disease based on their experience involving with vibrating equipment. In Malaysia, only limited work have done in the field of occupational vibration exposure since there is no legislation or rules to be follow accept the guideline especially in agriculture sector. The aims of the present work is to predict the HNS status of hand held grass cutter by taking into account the age, working experience and estimated hand-arm vibration exposure for both hand by using artificial neural network approach. This study will benefit a grass maintenance company's management about the information and instruction to ensure the health welfare of hand held grass cutter workers. 


\section{Predictive model}

Several predictive modelling approaches have been proposed over the past few years. In general, a linear modelling technique such as multiple regressions is the most common method used by researchers. Another option is artificial neural network able to solve nonparametric data which not yet extensively applied by human factor practitioner and ergonomics. However, there is still a research argument on the practitioner findings in the field of occupational safety and health $(\mathrm{OSH})$ study by means the type of prediction model should be selected due to the different answers published. There are many factors need to be considered in the selection of prediction manner which associated to processing the data itself. This selection is driven by the research objective and the specific case study data capabilities. In practice, the aims to develop prediction model is to maximize the accuracy of propose model and it is validity to be test in future [8]. Although, there are a number of limitations or uncontrolled parameter such as location, environment, human capacity made the model can only be test in certain sample area. For example, the effect of hand-arm vibration in Malaysia population should be different from other country, especially in the cases where the environment condition and the scope of work activities might be produce different data set. While, it is a positive advice to investigate the selected input factors for predictive model by each work unit activity. In OSH risk assessment, each work unit will expose to harmful event associated to the likelihood and severity of incident to happen. The selection of cause that induce to occupational diseases are being choose to develop predictive model, hence benefit to management if the model are appropriately used to improve productivity and worker health.

\subsection{Artificial neural network}

In general, ANN modelling was made up of highly interconnected nodes so called "neuron or unit". This interconnection is robust and store information in its connection through learning process which able to adjust and flexible in new environment adaption. The general neural network has been used by other researcher to employ feed forward multilayer back propagation learning approach $[9,10]$. For this type of learning processing methods a number of nodes which have independent connection between input layers, hidden layer and output layer have been recognized by the initial weight as shown in fig. 1 . Hidden layer consist of selected activation function, training rate, momentum rate, stopping criteria and others. In addition, model computation via incremental adjustments of weight was done by iteration process based on the minimum error calculation of each training cycle. Random starting point present as input training data set in the evaluation process. Hence, model post processing will be terminate as the minimum value of error been chosen by early stopping point which shows the mean square error (MSE) and regression (R) value. 


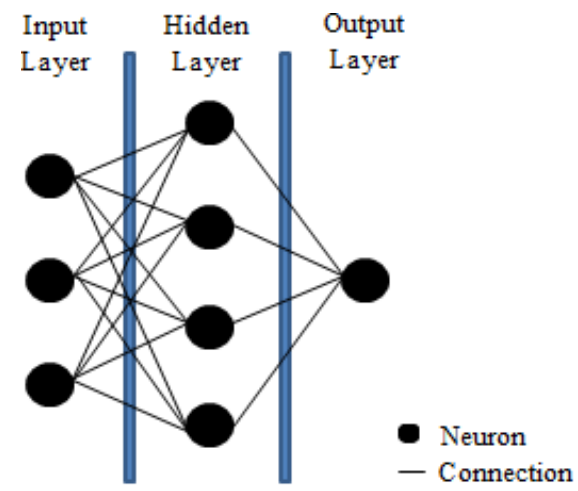

Figure 1: Basic structure of artificial neural network.

In the pre-processing techniques, data preparation is most crucial part. The data collected in the form of either interval or continuous variables produce parametric or non-parametric data distribution. Problem will be face if the data is not in the normal statistical distribution. Therefore, the normalization techniques have been proposed to minimize the standard deviation of data by reduces the interval span for each data, by means adjusting measured value [11]. There are several techniques in normalization such as standardized moment, coefficient of variation and feature scaling. In epidemiological study, combination between quantitative values collected from interview session and device measurement can give high interval of data value. However, the feature scaling techniques as shown in eqn (1) provide the input value in the range from 0 to 1 . Indeed, this technique proves to have better relation between targets and predicts value.

$$
x^{\prime}=\frac{x-\min (x)}{\max (x)-\min (x)}
$$

where $x^{\prime}$ is normalize input value between 0 and $1, x$ is the random actual data, $\min (x)$ is the minimum value of actual data and $\max (x)$ is maximum value of actual data.

ANN have successfully produced outcomes to a variety of function estimation and recognition problem (input-output, curve fitting, pattern classification, clustering, dynamic time series prediction, business forecasting, etc.). Problem that dealing with non-normal statistical distribution data which are indicate highly nonlinear variables relationships are suitable sample for ANN applications. In other point of view, the independent and dependent nonparametric estimation analysis of unknown model has been shown to applied multilayer feed forward network techniques [8]. For instance, multilayer feed forward network give a particular equation in the form of function, $f$ : where $f$ define the relationship agreement between independent $(x)$ and dependent $(y)$ variables, $f(x)=y$, hence the $y$ value able to determine any given value of $x$. Several studies have been done and the findings show that the ability of feed forward "sigmoid" architecture can estimate any continuous data [12, 13]. This 
unipolar form of "sigmoid" activation function shown in eqn (2) fit the random input values with the output range value from 0 to 1 .

$$
f(s)=\frac{1}{\left(1+e^{-s}\right)}
$$

where, $s$ is the nonlinear "sigmoid" activation function, $e$ is the exponential in mathematic formula. The best specific nonlinear activation function for back propagation network algorithm is the hyperbolic tangent function shown in eqn (3) which is the integration or bipolar sigmoid function; fit the input data in the range of -1 to 1 .

$$
\tanh (s)=\frac{\left(e^{s}-e^{-s}\right)}{\left(e^{s}+e^{-s}\right)}
$$

The activation function was added to the computing unit with the same kind of iteration process, hence; define a nonlinear weight in neuron connection [14]. The function will process until the desired weight input node define based on the minimum error value.

The post processing model indicates the MSE and R for the prediction model propose. The MSE compared between prediction and target value based on the eqn (4) while $\mathrm{R}$ define the relationship between the independent and dependent variables. In practice the value of MSE should be closed to " 0 " to produce high significant both hand prediction model for each of training, validate and testing set. In order to get give small value of MSE, the trial and error approach must been used by manipulating the parameter in hidden layer. Normally, the small number of MSE is associated with the R value near to " 1 " which defines the relationship between output and target data is strong.

$$
\operatorname{MSE}=\frac{1}{n} \sum_{i=1}^{n}\left(o_{i}-t_{i}\right)^{2}
$$

where, $n$ is the number of data, $o$ is the predicted value and $t$ is the target or output value after normalization.

\section{Methods}

\subsection{Sample population}

The current study investigation was conducted in central and southern part of peninsular Malaysia involving hand held grass cutter workers from subcontractor of council district and highway maintenance from August 2013 to November 2013. Total of 204 workers have been participated in this study. Majority of them are from foreign country whom able to speak and understand "Bahasa Malaysia". More than $45 \%$ of sample comes from Indonesia workers and the rest are from Bangladesh, Pakistan, India, Myanmar and local people as well. All of them are currently working in grass cutting operation to maintain grass growth either at highway, federal road, drain or building. Workers had the average of 5.5 hours working with operating grass cutter machine. All hand held grass 
cutter workers have been informed about this research and detail explanation regarding on the data collection process have been approved by company management.

\subsection{Quantitative observation and measurement}

Initially, the interviewed techniques have been used to determine the age, working experience and hand-arm vibration exposure per day. Parts of these independent variables were collected by using HAVS questionnaire adapted from vibration injury network [15]. In the end of the questionnaire, the picture of both hand consist of numerical value of each fingers phalange as shown in fig. 2, have been used to determine the HNS fingers colour changes. This technique needs professional judgments from the interviewer to select any phalanges fingers colour change. The interviewer has OSH background, and used his knowledge to investigate any fingers colour change. This observation was done after the grass cutter operation onsite and used for dependent variables in prediction model.
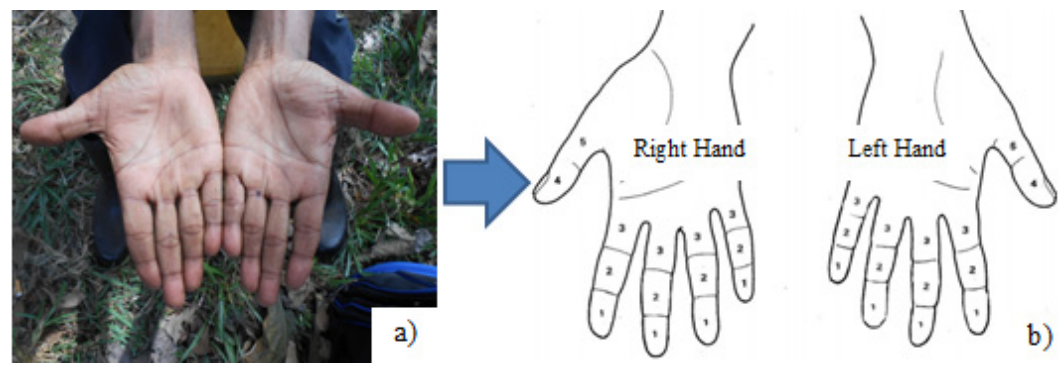

Figure 2: Effect of hand transmitted vibration on both hands a) onsite observations on fingers colour change; b) numerical scoring for each phalanges colour change.

It is necessary to determine also the hand transmitted vibration exposure during grass cutting operation. Hence, the 3 -axis accelerometer was attached following ISO 5349 [16] to both handle of hand held grass cutter shaft connected by wire to human vibration meter analyser (Larson Davis, HVM 100). This measurement techniques have been discuss on the other published manuscript [5]. The objective measurement will obtain the 3 axis vibration value transmitted to both hand of workers while in operation. Thus, the amplitude and frequency of vibration hazard were obtained, the results with the cumulative vibration magnitude were selected for estimation of hand-arm vibration exposure, $\mathrm{A}(8)$. The summary of independent and dependent variables have been shown in table 1. From OSH guidelines point of view, the mean value for estimated right and left hand vibration exposure have exceed the action limit according to European Directive on the risk of developing hand-arm vibration syndrome in the future [17]. 
Table 1: $\quad$ Summary of independent and dependent variables.

\begin{tabular}{|l|c|c|c|c|}
\hline \multicolumn{1}{|c|}{ Variables } & Mean & Min & Max & Std. deviation \\
\hline Age (years) & 32.05 & 15 & 56 & 9.294 \\
\hline Working experience (months) & 32.35 & 1 & 102 & 26.672 \\
\hline $\begin{array}{l}\text { Estimated right hand vibration } \\
\left.\text { exposure, A(8) (m/s }{ }^{2}\right)\end{array}$ & 5.309 & 1.3 & 12.6 & 2.609 \\
\hline $\begin{array}{l}\text { Estimated left hand vibration } \\
\left.\text { exposure, A(8) (m/s }{ }^{2}\right)\end{array}$ & 7.705 & 2.0 & 18.2 & 3.992 \\
\hline Right HNS & 8.12 & 0 & 20 & 4.154 \\
\hline Left HNS & 8.16 & 0 & 20 & 3.750 \\
\hline
\end{tabular}

\subsection{Prediction analysis}

The prediction model was developed by Matlab software version R2013a. Data produce from onsite collection consists of age, working experience, estimated vibration $\mathrm{A}(8)$ and $\mathrm{HNS}$ for both hand were selected as input and target, respectively. The data were exploring its normality by remove any outliers' data. After screening the data (pre-processing), the normalization technique took place. It minimize the range of data samples from value of 0.1 to 0.9 using the normalize equation. Hence, the random data were separated into 3 section which are $60 \%$ for training set, $20 \%$ for validate set and other $20 \%$ for testing set. For the ANN analysis, the number of hidden nodes was set as 4 after the trial and error processes which give the minimum value of error. The "tanh" activation function was set in hidden layer while for the output layer was used "purelinear". Further, the Lavenberg-Marquardt (trainlm) was used as the training data method due to fast learn ability. Other variables need to be set in ANN analysis approach such as, maximum number of epochs to train is 500, performance goal is $1 \mathrm{e}-4$, maximum validation failures is 50 , minimum performance gradient is $1 \mathrm{e}-10$ and maximum time to train in second is infinity. Finally, the performance index of the training, validate and test sets will depict in the form of R and MSE. The ANN architecture algorithm for develop prediction model shown in fig. 3 which consists of pre-processing, normalize, analysis and post processing techniques.

\section{Result and discussion}

The performance index for predicting HNS using age, working experience and estimated hand-arm vibration $\mathrm{A}(8)$ according to left hand and right hand were tabulated in table 2. The evaluation of training, validate and testing error function during training stage was found the minimum value of error by using the early stopping point method. Typically, the MSE for both hand are in excellent minimum error which are 1e-5. This shows that the development of ANN architecture has achieved the target to predict the HNS among hand held grass cutter. The training set give the minimum value of MSE compared to 


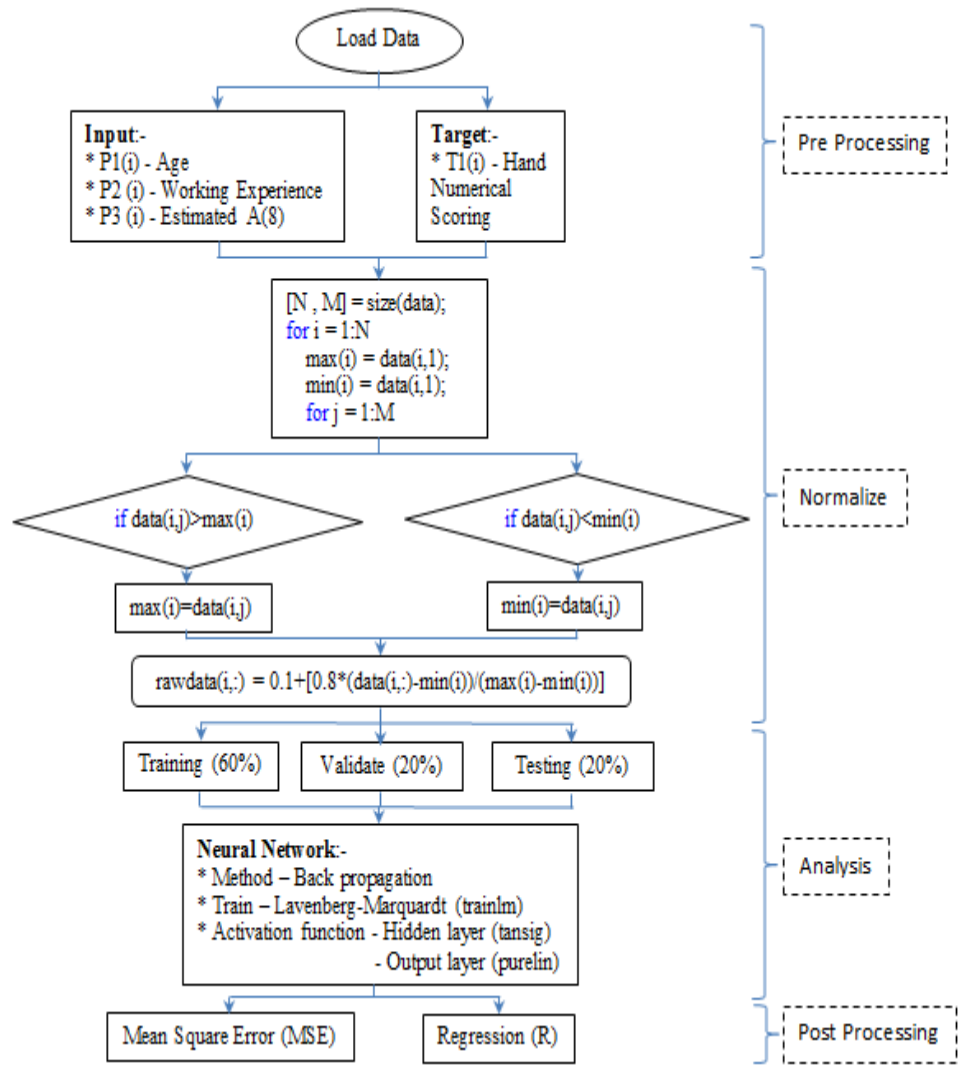

Figure 3: ANN architecture structure for developing prediction model.

validate and test set which the value is 7.344e-5 for the right hand and $4.7172 \mathrm{e}-5$ for the left hand respectively. The left hand training set shows less MSE indicating that the ANN algorithm is more suitable for the left hand. It happens due to different values in estimating vibration exposure $A(8)$ and the value of HNS for the left hand. However, the regression value is a better fit for the right hand since the value more approaches " 1 ". Although the regression value of the training set shows precise value near to " 0 ", the validate and test set give the value of 0.99916 and 0.99949 for the right hand, and 0.99862 and 0.99869 for the left hand. It can be seen that the value of the difference is very small, which is less than 0.001 . Thus, it indicates that the ANN architecture successfully develops the prediction model for HNS among grass cutter workers in Malaysia.

The combinations of all 3 data sets have produced results for $\mathrm{R}$ analysis as shown in fig. 4 for both hands. It is noted that the HNS regression value for the right hand is higher compared to the left hand which is 0.99937 and 0.99884 , respectively. Most of the data is tabulated linearly fit at the regression line equal 
Table 2: $\quad$ ANN performance index for training, validate and test sets.

\begin{tabular}{|l|l|l|l|}
\hline Performance Index & Training & Validate & Testing \\
\hline Right Hand & & & \\
\hline $\begin{array}{l}\text { Mean Square Error } \\
\text { (MSE) }\end{array}$ & $7.3444 \mathrm{e}-5$ & $9.1654 \mathrm{e}-5$ & $8.1063 \mathrm{e}-5$ \\
\hline Regression (R) & 0.99941 & 0.99916 & 0.99949 \\
\hline Left Hand & & & \\
\hline $\begin{array}{l}\text { Mean Square Error } \\
\text { (MSE) }\end{array}$ & $4.7172 \mathrm{e}-5$ & $8.0093 \mathrm{e}-5$ & $6.4748 \mathrm{e}-5$ \\
\hline Regression (R) & 0.99902 & 0.99862 & 0.99869 \\
\hline
\end{tabular}

to " 1 ". The left hand diagram shows the overlapping point at the area target 0.6 and 0.2 . This indicate that the majority of hand held grass cutter subjects exposed to vibration induce white fingers more dominant compared to the right hand as the mean value for HNS is higher. However, both hands obtained the best regression prediction model as the output can be assumed as predicting and the target is the input variables of the model. The difference of prediction model for both hands is only 0.03 for constant regression coefficient while the intersection point is 0.0154 , respectively. The result from this finding propose that there are significant health effects of hand-arm vibration exposure for both hands using non-linear activation function of HNS.
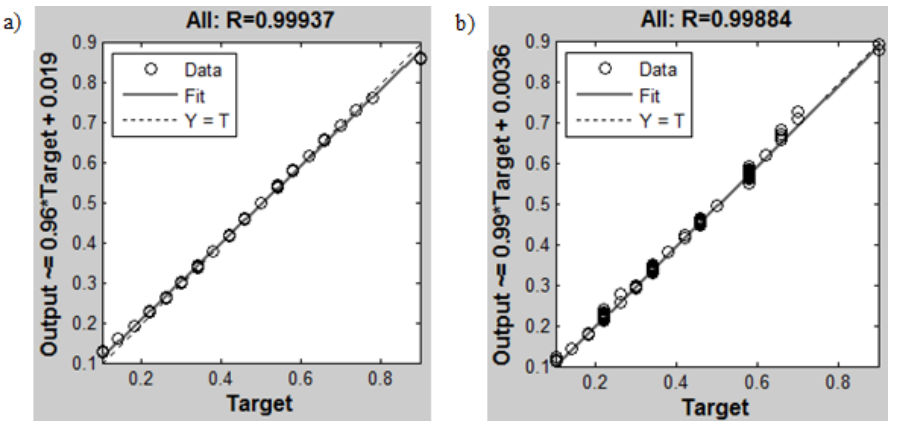

Figure 4: The results of regression analysis carried out on the all set of data. a) right hand b) left hand.

Also in this study, the ANN performance was evaluated using relative error when compared the predicted and the actual data. As can be seen from table 3, the ANN was train successfully and produces the small number of relative error of less than 0.01 for example of three data. To ensure correct training, the ANN predicted result was test using the actual data, for which the relative error was obtained using eqn. (5). Hence, the network develop here is expected to be suitable to determine the effect of HNS among hand held grass cutter workers based on the age, working experience and estimated hand-arm vibration $\mathrm{A}(8)$. 
Table 3: A comparison of the HNS results and the real HNS data for training set (example 3 data).

\begin{tabular}{|l|c|c|c|}
\hline \multicolumn{1}{|c|}{ No } & $\begin{array}{c}\text { ANN } \\
\text { predicted }\end{array}$ & $\begin{array}{c}\text { Actual } \\
\text { data }\end{array}$ & $\begin{array}{c}\text { Relative } \\
\text { error }\end{array}$ \\
\hline Right hand & & & \\
\hline 1 & 11.97 & 12 & 0.0025 \\
\hline 2 & 2.97 & 3 & 0.0100 \\
\hline 3 & 6.42 & 6 & -0.0700 \\
\hline Left hand & & & \\
\hline 1 & 12.71 & 12 & 0.0590 \\
\hline 2 & 5.98 & 6 & 0.0033 \\
\hline 3 & 5.99 & 6 & 0.0017 \\
\hline
\end{tabular}

The recommended point to the hand held grass cutter company, is to reduce the hand-arm vibration exposure by minimizing the machine contact hour and prepare standard maintenance operation procedure to avoid the vibration increase due to unbalance of rotating machine. This study of found that, majority of the workers already undergo stage 2 of vascular and sensori-neural disease. Perhaps, the company management should aware of their workers' health affected by work activity. According to our understanding, there is a responsibility and accountability of the company whom provided the workers job to ensure the safety, health and welfare of employee. So, the propose prediction model can be used by company management to select and predict the health condition of hand held grass cutter workers. Further study need to compare this network again other work activity such as the grinding worker and logging workers due to the different magnitude of vibration transmitted.

$$
\mathrm{HNS}=\frac{H N S_{\text {actual }}-H N S_{\text {predicted }}}{H N S_{\text {actual }}}
$$

\section{Conclusion}

It can be concluded that the hand transmitted vibration exposure were exceed the permissible limit for both hand. Hence, grass cutter workers suffer from decreased hand function ability due to their occupational operation. Based on the epidemiological study data, the developed ANN architecture have obtain the best performance index indicated by the value of MSE which closed to " 0 " while R value is closed to " 1 ". Thus, the prediction model of HNS among hand held grass cutter worker in Malaysia have been proposed and benefited to workers and company management to protect against hand-arm vibration exposure by determine their age, working experience and estimated vibration exposure, $A(8)$. It is also suggested for the hand held grass cutter workers to undergo health surveillance supervised by occupational health doctor periodically at least once a year to avoid permanent hand disability. 


\section{Acknowledgements}

This study was supported by the Research and Development (R\&D) grant under the National Institute of Occupational Safety and Health, Malaysia. The authors gratefully thank the cooperation given by the leading Malaysia highway maintenance company management ant all the grass cutter workers that took part in this work.

\section{References}

[1] Bovenzi, M., Pinto, I., Picciolo, F., Mauro, M. \& Ronchese, F., Frequency weightings of hand-transmitted vibration for predicting vibration-induced white finger. Scandinavian Journal of Work, Environment \& Health, 37(3), pp. 244-252, 2011.

[2] Inyang, N. \& Al-Hussein, M., Ergonomic hazard quantification and rating of residential construction tasks. Annual Conference of the Canadian Society for Civil Engineering, Canada, pp. 1885-1895, 2011.

[3] Bovenzi, M., Exposure-response relationship in the hand-arm vibration syndrome: an overview of current epidemiology research. International Archives of Occupational and Environmental Health, 71(8), pp. 509-519, 1998.

[4] Gemne, G., Pyykko, I., Taylor, W. \& Pelmear, P.L., The Stockholm Workshop scale for the classification of cold-induced Raynaud's phenomenon in the hand-arm vibration syndrome (revision of the TaylorPelmear scale). Scandinavian Journal of Work, Environment \& Health, 13, pp. 275-278, 1987.

[5] Azmir, N.A., Ghazali, M.I. \& Yahya, M.N., Hand arm vibration and personal noise exposure characteristics of hand held grass cutter machines. Proc. of the $21^{\text {st }}$ International Congress on Sound and Vibration, Beijing, 2014.

[6] Bylund, S.H. \& Burstrom, L., The influence of gender, handle size, anthropometric measures, and vibration on the performance of a precision task. International Journal of Industrial Ergonomics, 36(10), pp. 907914, 2006.

[7] McCallig, M., Paddan, G., Van Lente, E., Moore, K. \& Coggins, M., Evaluating worker vibration exposure using self-reported and direct observation estimates of exposure duration. Applied Ergonomics, 42(1), pp. 37-45, 2010.

[8] Agha, S. \& Alnahhal, M., Neural network and multiple linear regression to predict school children dimensions for ergonomic school furniture design. Applied Ergonomics, 43(6), pp. 979-984, 2012.

[9] Zhang, B., Horvath, I., Molenbroek, J.F.M. \& Snijders, C., Using artificial neural networks for human body posture prediction. International Journal of Industrial Ergonomics, 40(4), pp. 414-424, 2010. 
[10] Bakhshandeh Amnieh, H., Mozdianfard, M.R. \& Siamaki, A., Predicting of blasting vibrations in Sarcheshmeh copper mine by neural network. Safety Science, 48(3), pp. 319-325, 2010.

[11] Wu, J.Z., Dong, R.G., Welcome, D.E. \& Xu, X.S., A method for analysing vibration power absorption density in human fingertip. Journal of Sound and Vibration, 329(26), pp. 5600-5614, 2010.

[12] Reddy, C.S. \& Raju, K., A concise neural network model for estimating software effort. International Journal of Recent Trends in Engineering, 1(1), pp. 188-193, 2009.

[13] Taha, Z., Grip strength prediction for Malaysian industrial workers using artificial neural networks. International Journal of Industrial Ergonomics, 35(9), pp. 807-816, 2005.

[14] Rojas, R., Neural Network: A Systematic Introduction, Springer-Verlag: Berlin, 1996.

[15] Griffin, M. \& Bovenzi, M., The diagnosis of disorders caused by handtransmitted vibration: Southampton Workshop 2000. International archives of occupational and environmental health, 75(1-2), pp. 1-5, 2002.

[16] BS EN ISO 5349-2, Mechanical vibration - measurement and evaluation of human exposure to hand-transmitted vibration - Part 2: Practical guidance for measurement at the workplace. British Standard, 2002.

[17] Directive 2002/44/EC of the European Parliament and the Council of $25^{\text {th }}$ June 2002 on the minimum health and safety requirement regarding the exposure to workers to the risks arising from physical agents (vibration). The Official Journal of the European Communities, 45(EC), pp. 13-19, 2002. 